\title{
APPROXIMATE DESCRIPTION OF DECAYING QUASI-STATIONARY STATE
}

\author{
A. Matulis ${ }^{a}$ and G. Kiršanskas ${ }^{b}$

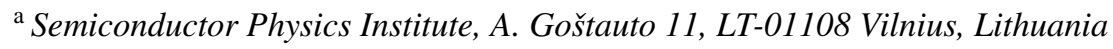 \\ E-mail: amatulis@takas.lt \\ ${ }^{\mathrm{b}}$ Department of Theoretical Physics, Vilnius University, Sauletekio 9, LT-10222 Vilnius, Lithuania
}

Received 9 July 2009; revised 22 September 2009; accepted 18 December 2009

\begin{abstract}
A simple model of the decaying quantum state composed of a one-dimensional electron moving in the potential with the quantum dot separated from the infinite half-axis by the $\delta$-function is studied. The quasibound quantum dot state properties calculated by means of approximate methods (complex energy technique and local density of states method) are compared with results obtained with the aid of an exact analytical non-stationary wave function. The physical meaning of the emitted electron wave function peculiarities is discussed.
\end{abstract}

Keywords: quasi-bound state, decay, lifetime, local density of states

PACS: 03.65.-w, 73.21.La

\section{Introduction}

Progress in nanometre technology has triggered a broad activity in low-dimensional quantum systems [1]. During the last five years the interest has shifted to graphene, [2,3] the single layer of graphite that is an ideal two-dimensional system. It is expected that this material will serve as a base for new electronic and optical devices. Charge carriers in graphene behave like "relativistic" massless particles, and due to the socalled Klein effect [4] the control of their behaviour by means of electromagnetic fields is one of the most challenging tasks. This situation occurs because the quantum dots in graphene have no bound states and the electrons escape from them sooner or later. Thus, the description of quasibound states becomes of importance. For this purpose various approximate methods are used, mostly those considering some approximate stationary problem. One of them is the so-called complex energy technique [5] (see also [6]), which relates the imaginary part of energy eigenvalue with the inverse lifetime of quasibound state. The other way to determine the above lifetime is to extract it from the width of resonances in the so-called local density of states of an artificially confined system [7, 8]. The quasi-classical approach [9] for the description of the quasibound states is also used.
The aim of this paper is to evaluate the advantages and shortcomings of various approximate quasibound state description methods comparing the results with the exact solution for some analytically solvable model of a decaying quantum state, which is an example of most simple non-stationary problem. For this purpose we use the model of one-dimensional (1D) quantum system where the electron moves in the potential with a repulsive $\delta$ function that separates the quantum dot from the infinite half $x$ axis. This model has been examined by a number of people, in particular, by Petzold and Winter [10], and more recently by van Dijk [11-13] mostly in relation with the problem of radioactive decay of nucleus.

The paper is organized as follows. In Section 2 the model is introduced and the exact solution is presented. The peculiarities of the wave function of the quasibound states are discussed in Section 3 paying attention to the pole and saddle contributions. In the next three sections the approximate methods - complex energy technique, local density of states method, and the quasi-classical approach - are given and their result is compared with the exact analytical solution. The conclusions are given in Section 7, and in Appendix the simple derivation of the wave function of the decaying state is proposed. 


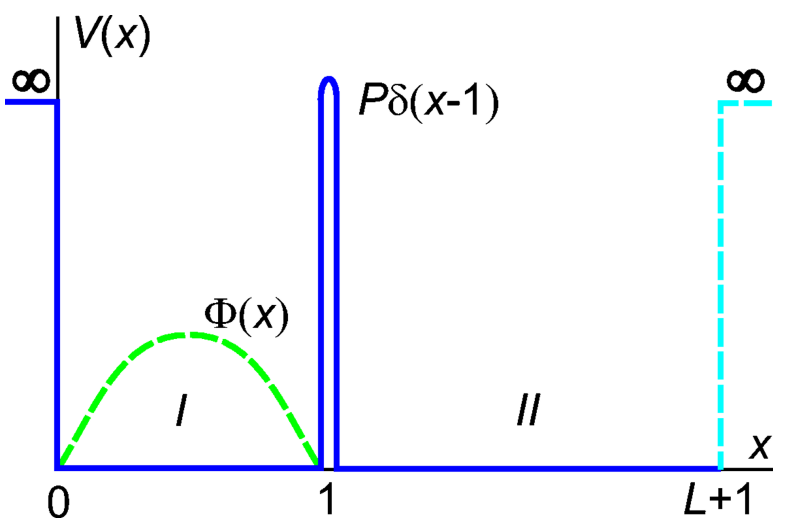

Fig. 1. Potential.

\section{Model}

We consider 1D electron moving in a potential

$$
V(x)=P \delta(x-1)
$$

shown in Fig. 1 by solid (blue on-line) curve. We restrict our consideration to the positive half-axis $(x>0)$. That is why there is a hard wall at the origin $(x=0)$. The Dirac function at the point $x=1$ makes the quantum dot in the region $I(0<x<1)$ separating it from the infinite region $I I(1<x<\infty)$. For the sake of simplicity we shall use the dimensionless units measuring all distances in the widths $a$ of this quantum dot, the energy in $\varepsilon_{0}=\hbar^{2} / m a^{2}$ units, and time in $\hbar / \varepsilon_{0}$ units.

Due to the penetrability of the narrow $\delta$-type barrier the quantum dot has no stationary states. Nevertheless, if the the barrier is high enough $(P \gg 1)$, the tunnelling of electron outside the dot is weak, and there are socalled quasibound states. The self-consistent description of such state follows from the solution of the time dependent Schrödinger equation

$$
\mathrm{i} \frac{\partial}{\partial t} \Psi(x, t)=H \Psi(x, t)
$$

with the Hamiltonian

$$
H=H_{0}+V(x)=-\frac{1}{2} \frac{\partial^{2}}{\partial x^{2}}+V(x)
$$

and hard wall boundary condition

$$
\Psi(0, t)=0 .
$$

As we are going to solve the non-stationary problem the above Schrödinger equation has to be supplemented by some initial condition. For this purpose we use the wave function of the ground state of the isolated quantum dot $(P=\infty)$ that reads

$$
\Phi(x)=\sqrt{2} \sin (\pi x)
$$

and is shown in Fig. 1 by dashed (green on-line) curve.
So, we assume that at the initial moment $t=0$ we cut the top of the Dirac function by fixing it with $P<\infty$. Then electron starts to escape from the quantum dot, and we describe this process by solving Eq. (2) in the time interval $0<t<\infty$ with the initial condition (5).

According to Refs. [11-13] the solution of this problem can be presented as the integral in the complex momentum $k=u+\mathrm{i} v$ plane (see the simple derivation in Appendix):

$$
\begin{aligned}
& \Psi(x, t)=\frac{1}{\mathrm{i} \pi} \int_{-\infty}^{\infty} \mathrm{d} k \frac{F(k)}{Q(k)} \mathrm{e}^{-\mathrm{i} k^{2} t / 2} \\
& \times\left\{\mathrm{e}^{\mathrm{i} k x}+\frac{P}{\mathrm{i} k} \Theta(x-1)\left[\mathrm{e}^{\mathrm{i} k x}-\mathrm{e}^{\mathrm{i} k(x-2)}\right]\right\},
\end{aligned}
$$

where $F(k)$ is given by Eq. (A12) and

$$
\begin{aligned}
& Q(k)=\left(\cos k+\frac{2 P}{k} \sin k\right)^{2}+\sin ^{2} k \\
& =\frac{P^{2}}{k^{2}}\left(\mathrm{e}^{2 \mathrm{i} k}-1+\frac{\mathrm{i} k}{P}\right)\left(\mathrm{e}^{-2 \mathrm{i} k}-1-\frac{\mathrm{i} k}{P}\right) .
\end{aligned}
$$

Integrating the above wave function in the quantum dot (in region $I$ ) we obtain the survival amplitude

$$
\begin{aligned}
w(t) & =\int_{0}^{1} \mathrm{~d} x \Phi(x) \Psi(x, t) \\
& =\frac{1}{\pi} \int_{-\infty}^{\infty} \mathrm{d} k \frac{F^{2}(k)}{Q(k)} \mathrm{e}^{-\mathrm{i} k^{2} t / 2}
\end{aligned}
$$

and the quantity

$$
W(t)=|w(t)|^{2}
$$

that gives the probability to find the electron in the initial state which in the case of small barrier penetrability $(P \gg 1)$ coincides with the probability to find the electron in the dot [14].

Due to the factorization of $Q(k)$ function the expression of the wave function outside the dot (in region $I I$ ) can be presented in more simple form:

$$
\Psi(x, t)=\frac{1}{\pi P} \int_{-\infty}^{\infty} \frac{\mathrm{d} k k F(k) \mathrm{e}^{\mathrm{i}\left(k x-k^{2} t / 2\right)}}{\mathrm{e}^{2 \mathrm{i} k}-1+\mathrm{i} k / P}
$$




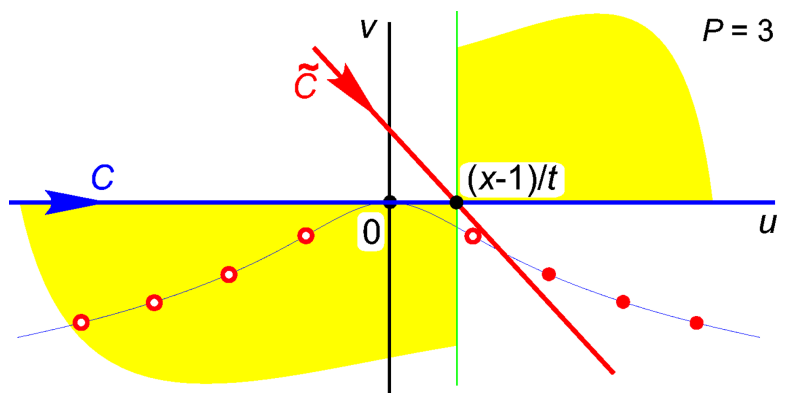

Fig. 2. Complex $k$ plane and the contours for integration of integral (10)

\section{Wave function outside the dot}

The convergence of complex integral (10) depends on the exponent in integrand (including the exponent in function $F(k)$ ) whose argument can be rewritten as

$f(k)=\frac{k^{2} t}{2}-k(x-1)=\frac{t}{2}\left(k-\frac{x-1}{t}\right)^{2}-\frac{(x-1)^{2}}{2 t}$.

The sign of the first term indicates that there are two quadrants (in respect of point $k_{0}=(x-1) / t$ ) in the complex $k$-plane (shaded (yellow on-line) in Fig. 2) where the integral does not converge. Thus, the best convergence is achieved by replacing the initial horizontal integration contour $C$ by the contour $\tilde{C}$ shown in Fig. 2 by the (red on-line) solid line coinciding with the bisector of the fourth quadrant and passing through the saddle point $k_{0}$. Transforming the integration contour, however, we have to take into account the contribution of poles that are crossed during this transformation. As the function $F(k)$ has no poles only the zeros of the integrand denominator matter. They are defined by solving equation

$$
\mathrm{e}^{2 \mathrm{i} k}=1-\frac{\mathrm{i} k}{P}
$$

Separating the real and imaginary parts of it

$$
\begin{aligned}
\mathrm{e}^{-2 v} \cos (2 u) & =1+\frac{v}{P}, \\
\mathrm{e}^{-2 v} \sin (2 u) & =-\frac{u}{P},
\end{aligned}
$$

we easily define the curve

$$
u=P \sqrt{\mathrm{e}^{-4 v}-\left(1+\frac{v}{P}\right)^{2}},
$$

and zeroes of Eq. (12) solving numerically Eq. (13a) along the curve (14). They are shown in Fig. 2 by (red on-line) circles. We see that during the contour transformation some of these points in the fourth quadrant (shown as full circles) are intersected. Thus, calculating the wave function integral (10) the contribution of the corresponding integrand poles

$$
\Psi_{n}(x, t)=\frac{\pi \sqrt{2} k_{n}^{2} \mathrm{e}^{\mathrm{i}\left[k_{n}(x-1)-k_{n}^{2} t / 2\right]}}{P\left(\pi^{2}-k_{n}^{2}\right)\left(1+2 P-2 \mathrm{i} k_{n}\right)}
$$

has to be taken into account together with the saddle point contribution (the integral calculated along the contour $\tilde{C}$ ). The pole contribution (15) gives the characteristic exponential behaviour of the wave function.

In the case of weak tunnelling $(P \gg 1)$ the position of poles (namely, the solution of Eq. (12)) can be obtained by expansion in $P^{-1}$ powers, and it leads to

$$
k_{n}=\pi n\left(1-\frac{1}{2 P}-\frac{\mathrm{i} \pi n}{4 P^{2}}\right),
$$

where $n$ is an integer number nonequal to zero.

We see that the small tunnelling probability shifts the poles corresponding to the real eigenvalues of the isolated quantum dot $\pi n$ into the lower complex halfplane. It is also evident that the number of poles contributing to the wave function integral depends on the values of parameter $(x-1) / t$. Namely, enlarging $x$, we exclude step by step the contributions of poles.

Due to real negative exponent argument along the transformed contour $\tilde{C}$ the saddle point contribution can be easily estimated numerically with any desired accuracy. As the choice of the integration contour $\tilde{C}$ along the bisector of the fourth quadrant is quite arbitrary the separation of the saddle and pole contributions is rather provisory. Nevertheless, it is rather useful for the interpretation of processes ongoing in the quantum dot.

The real part of the exact wave function (10) including all saddle and pole contributions is shown in Fig. 3 for $P=3$, which is the case of small tunnelling probability. The imaginary part of it looks similar, except some non-essential phase shift. We see two characteristic electron wave function features. First, there are well expressed wave crests along the direction shown by (blue on-line) dashed line. It corresponds to equation $x=v_{f} t$, where

$$
v_{f}=\varepsilon / k=k / 2 \approx 1.39
$$

coincides with the phase velocity of the electronic wave, corresponding to the momentum $k$ of the ground state of the dot. The next evident feature is that the main pulse of emitted electron wave moves faster. Its motion is indicated by the (red on-line) solid line $x=v_{g} t$ where $v_{g}=\mathrm{d} \varepsilon / \mathrm{d} k=k$ coincides with the group velocity. 


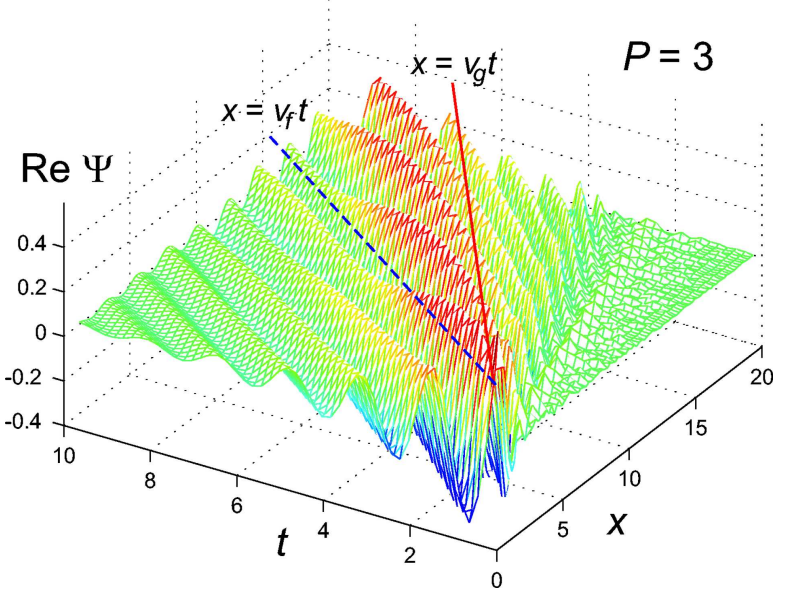

Fig. 3. The real wave function component outside the dot.

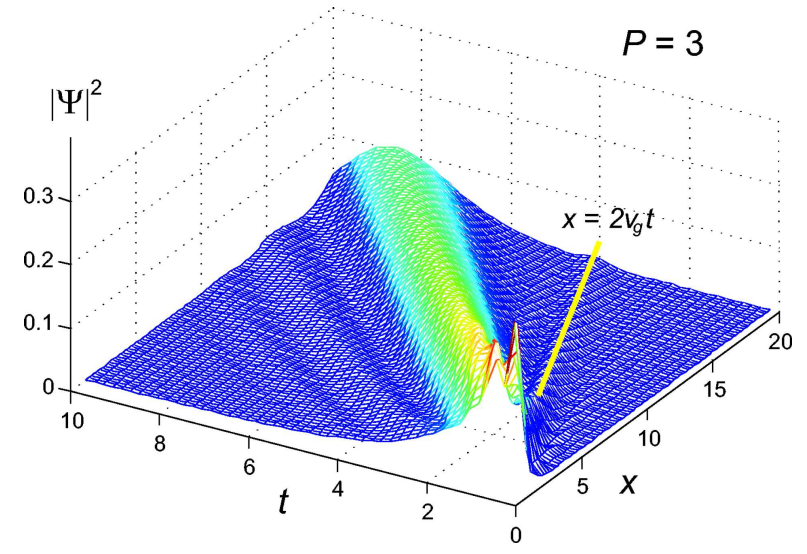

Fig. 4. Modulus of the wave function squared outside the dot.

Thus, the vacuum is a rather dispersive media for the classical electron.

From now on we shall not pay attention to the wave function's phase, restricting the consideration to the wave function modulus squared corresponding to the probability to find electron in a given point $x$. That is why instead of Fig. 3 we shall plot a more simple Fig. 4. Although this plot is much more regular, nevertheless we see clearly some waving in front and after the main pulse that is indicated by the bright stripe. This waving has to be related to the initial condition. The matter is that by cutting off the barrier (see the discussion in Sec. 2) at the initial moment $t=0$ we perturb the system, and as a result all electron states become excited. Consequently, the dot emits the electron from all these excited states with the corresponding group velocities $k_{n} \approx \pi n$. The electron pulse emitted from the first excited state $(n=2)$ can be clearly seen in Fig. 4 (indicated by the (yellow on-line) solid line).

This electron emission from various excited states is seen more clearly in Fig. 5 where the pole contributions to wave function (15) are plotted. Here we see

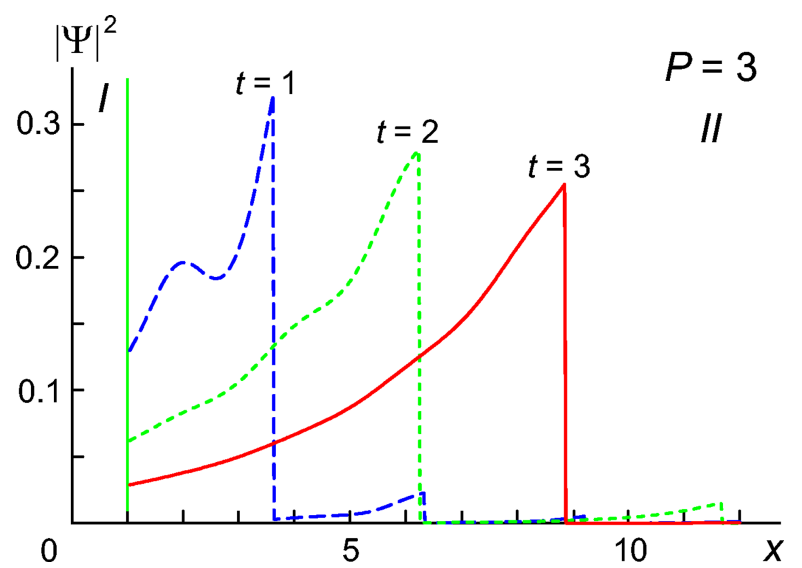

Fig. 5. The coordinate dependence of the electron wave function modulus squared outside the dot at various time moments including only the pole contribution.

the vertical wavefronts moving from the dot with the above group velocities. The wavefronts corresponding to the electron emission from the ground state, the first excited state, and even from the second one are seen.

Note there are well resolved oscillations after the main pulse in the region of small times gradually disappearing when the time grows up. In quantum mechanics, however, there are no sharp pulses nor wavefronts due to known smearing of wave packets, which is inherent to any wave process. So it is evident that the saddle contribution is of importance. It is confirmed by Fig. 6 where the wave function with both, saddle and pole contributions included, is shown. We see that the saddle contribution is important in region $I I$, especially close to the main emission pulse. This saddle contribution smears the wavefronts, although it does not change the picture qualitatively, and the pulses corresponding to the electron emission from the ground and excited levels are quite distinguishable.

In order to reveal the physical meaning of the

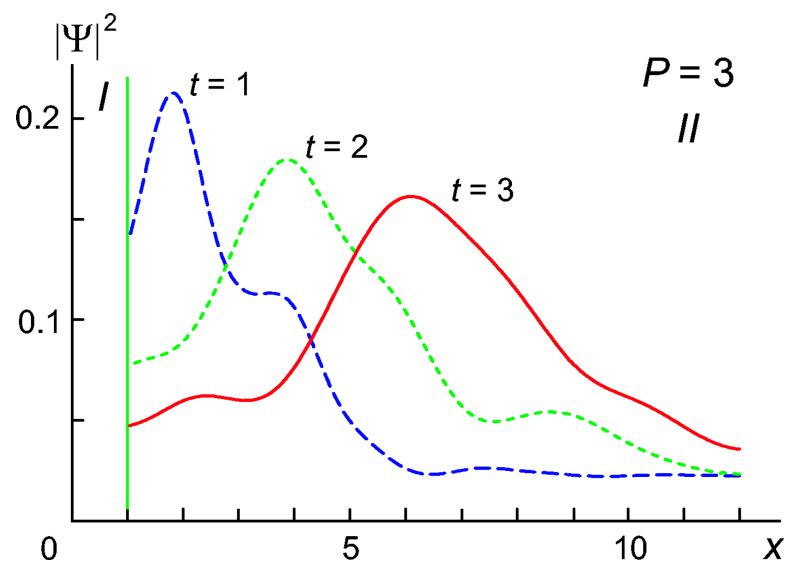

Fig. 6. The same as in Fig. 5 but with both saddle and pole contributions included. 


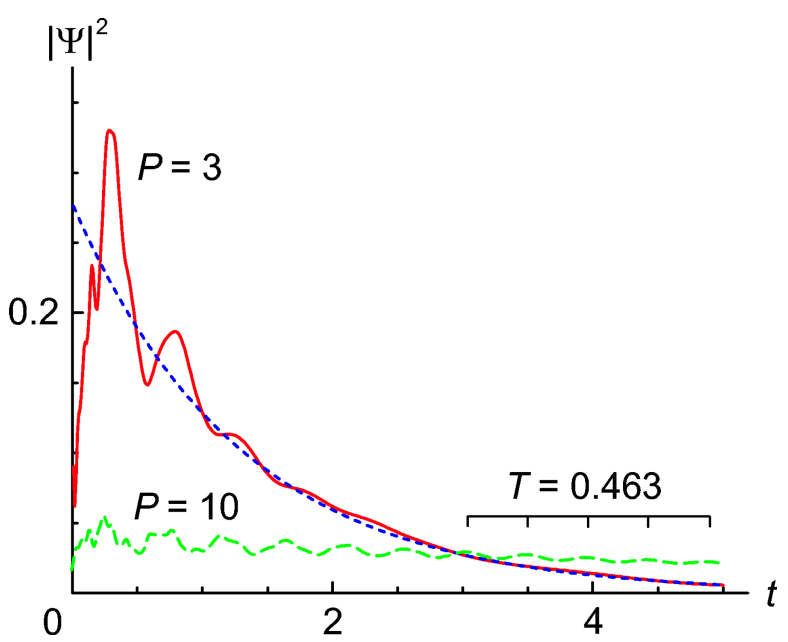

Fig. 7. The time dependence of the wave function at the quantum dot edge.

oscillations following the main pulse we calculated the wave function values at the quantum dot edge $(x=1)$. They are shown in Fig. 7. These oscillations at the quantum dot edge can be considered as an origin of the above waving of the wave function outside the dot following the main electron emission pulse. In the case of $P=3$ the quantum dot edge wave function oscillations decay rather fast with time, and the wave function modulus squared approaches the (blue on-line) dotted curve which indicates the first pole contribution. The oscillations are pronounced much better for less penetrable barrier as it is seen in the case of $P=10$ shown by (green on-line) dashed curve. It is remarkable that in the asymptotic region $(t \rightarrow \infty)$ they convert into sinusoidal oscillation with the period $T_{0}=0.463$. This period coincides pretty well with the period of beats appearing due to the interference of the ground and first excited states' wave functions:

$$
|\Psi|^{2} \sim\left|\mathrm{e}^{-\mathrm{i} \varepsilon_{1}} \psi_{1}+\mathrm{e}^{-\mathrm{i} \varepsilon_{0}} \psi_{0}\right|^{2} \rightarrow \cos \left[\left(\varepsilon_{1}-\varepsilon_{0}\right) t\right]
$$

with $T=2 \pi /\left(\varepsilon_{1}-\varepsilon_{0}\right) \approx 0.470$. Thus, the wave function outside the dot is quite sensitive to the details of initial state preparation. It demonstrates not only the emission from all excited states, but also the interference of their wave functions.

Finishing the discussion about the exact solution of the non-stationary Schrödinger equation let us say some words about the electron behaviour in the dot. The calculation of complex integral (8) defining the survival amplitude and finally the probability to find the electron in the dot is similar to the calculation of Eq. (10) that was just considered. It is even more simple, because the argument of exponent in the integrand does not depend on the coordinate $x$. The single difference

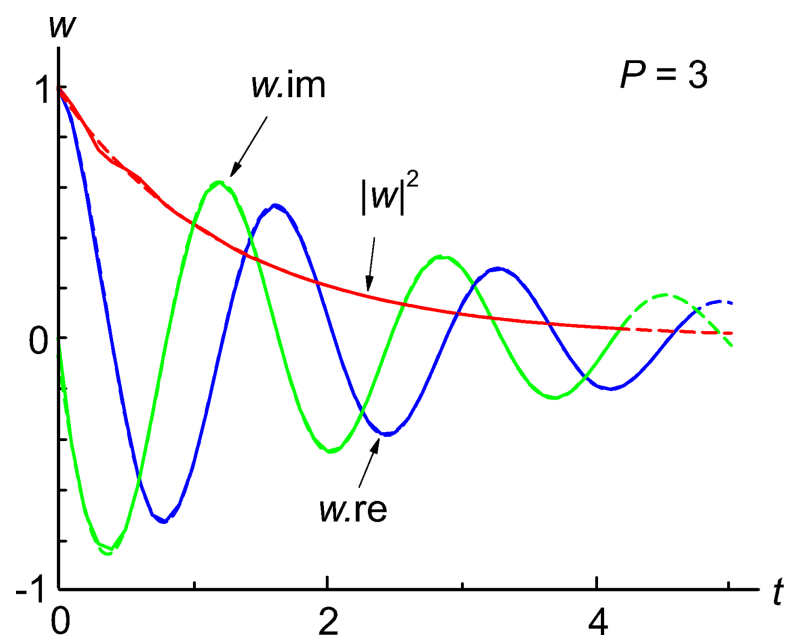

Fig. 8. Survival amplitude (8): solid curves represent the exact solution, dashed curves the first pole contribution.

is that the function $Q(k)$ has twice more zeroes as compared with the function in denominator of integral (10). The additional poles can easily be obtained by means of reflection $v \rightarrow-v$. The result for survival amplitude in the case of $P=3$ is shown in Fig. 8 by solid curves. The contribution of the first pole is shown by the dashed curves. The coincidence of solid and dashed curves allows to conclude that the behaviour of the electron in the dot, namely, the survival amplitude, the energy of the state, and the lifetime are described satisfactorily by the single pole approximation in contrary to the function outside the dot where the poles corresponding to the excited states and the saddle contribution are important.

\section{Complex energy method}

Now we switch our attention to the main purpose of our consideration and discuss the approximate methods.

We start with the most simple one - the complex energy method. This method is based on the assumption that the non-stationary process of decay can be described by some fictitious stationary problem with complex energy eigenvalue. The Schrödinger equation is solved in the dot assuming the radiative boundary condition at its edge, namely, with the assumption that in the region $I I$ the wave function is composed of a single outgoing exponent. In the electrodynamics it is known as a Sommerfeld radiation boundary condition [15].

So inserting the wave function

$$
\Psi(x, t)=\mathrm{e}^{-\mathrm{i} E t} \Psi(x)
$$

into Eq. (2), we arrive at the eigenvalue problem

$$
\{H-E\} \Psi(x)=0 .
$$


The solution of this equation is similar to the solution presented in Appendix where we were looking for the complete set of eigenfunctions. The only difference is that now the wave function outside the dot is composed of a single outgoing exponent. Thus, the solution of Eq. (20) reads

$$
\Psi(x)=\left\{\begin{array}{l}
A \sin (k x), 0<x<1, \\
B \mathrm{e}^{\mathrm{i} k(x-1)}, 1<x<\infty .
\end{array}\right.
$$

Applying boundary conditions (A6) we arrive at the following set of algebraic equations:

$$
\begin{gathered}
A \sin k=B, \\
\mathrm{i} k B-k A \cos k=2 P A \sin k .
\end{gathered}
$$

Finally, zeroing its determinant we obtain the dispersion equation (12). Consequently, the eigenvalues of complex momentum coincide with the poles shown in Fig. 2. In the case of weak tunnelling they are given by Eq. (16). Thus, we obtain the following complex energy eigenvalues:

$$
E_{n}=E_{n}^{(0)}-\mathrm{i} \Gamma_{n}=\frac{1}{2} k_{n}^{2}=\frac{1}{2}\left(u_{n}^{2}-v_{n}^{2}\right)+\mathrm{i} u_{n} v_{n} .
$$

It is evident that only filled (red on-line) circles in the fourth quadrant have to be taken into account, as the zeroes in the third quadrant lead to the wave function growing in time that has no physical sense.

Finally the result obtained by the complex energy technique is shown in Fig. 9. The positions of quasibound states and their widths are shown as a function of barrier height $P$.

Substituting the eigenvalue $k_{n}$ into Eq. (21) we obtain the corresponding eigenfunction.

$$
\begin{aligned}
& \left|\Psi_{n}(x)\right|^{2}=\frac{1}{2}\left|A_{n}\right|^{2} \\
& \times\left\{\begin{array}{cc}
\cosh \left(2 v_{n} x\right)-\cos \left(2 u_{n} x\right), & 0<x<1, \\
{\left[\cosh \left(2 v_{n} x\right)-\cos \left(2 u_{n} x\right)\right] \mathrm{e}^{2 v_{n}(x-1)}} & 1<x<\infty .
\end{array}\right.
\end{aligned}
$$

The modulus of the ground state wave function squared is shown in Fig. 10. It resembles the wave function plots presented in Fig. 5, what is quite natural because the complex energy method coincides with the one pole approximation. Of course there are no wavefronts nor pulses, and no fine structure. It allows us to conclude that the complex energy method can hardly be useful

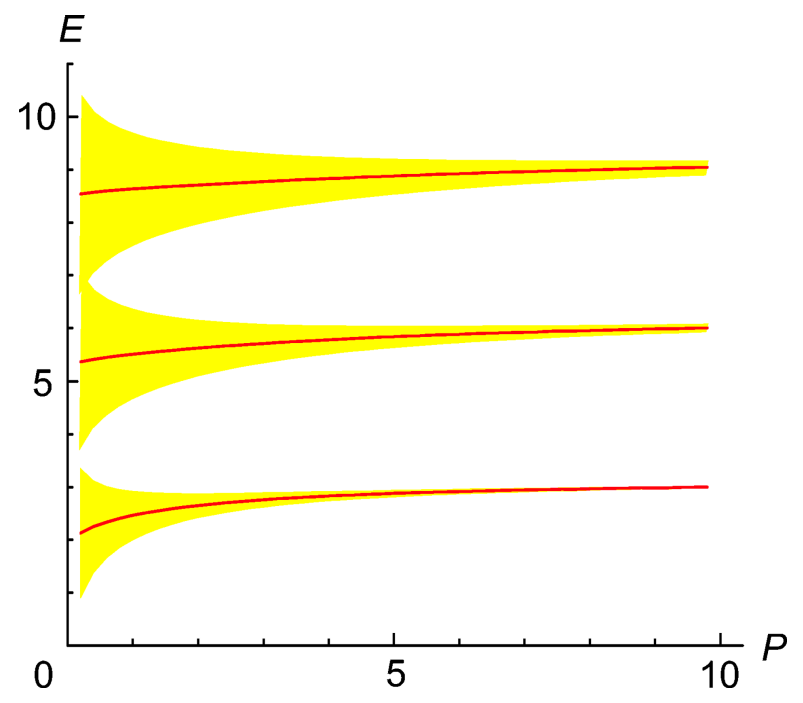

Fig. 9. Quasi-bound states as functions of the barrier height $P$ : solid (red on-line) curves mark the energy of the state and the widths are shown as (yellow on-line) faded regions.

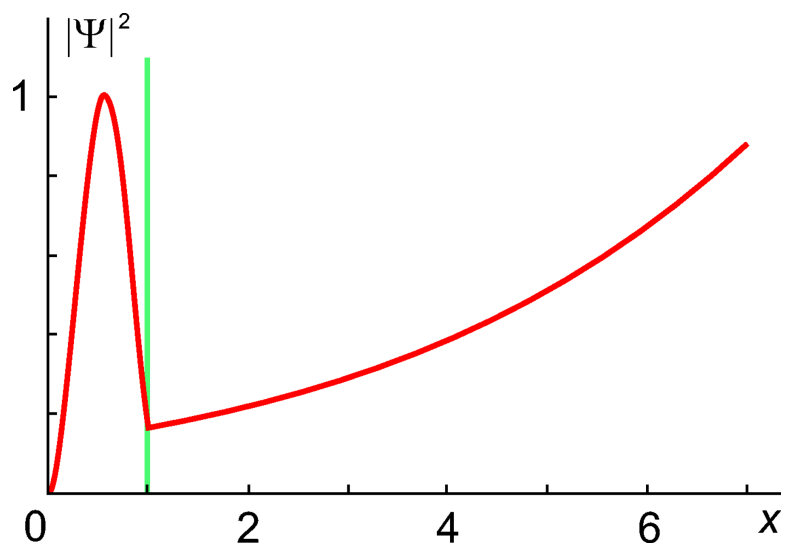

Fig. 10. Modulus of the wave function squared, obtained by the complex energy method.

for the wave function calculation of the decaying quasibound state. However, it gives us reasonable average characteristics of this state, such as approximate energy and lifetime.

\section{Local density approximation}

The density of states, or the number of available electron states per unit energy plays an important role in description of various quantum effects. Formally its mathematical definition reads:

$$
\rho(\varepsilon, x)=\sum_{n} \delta\left(\varepsilon-E_{n}\right)
$$




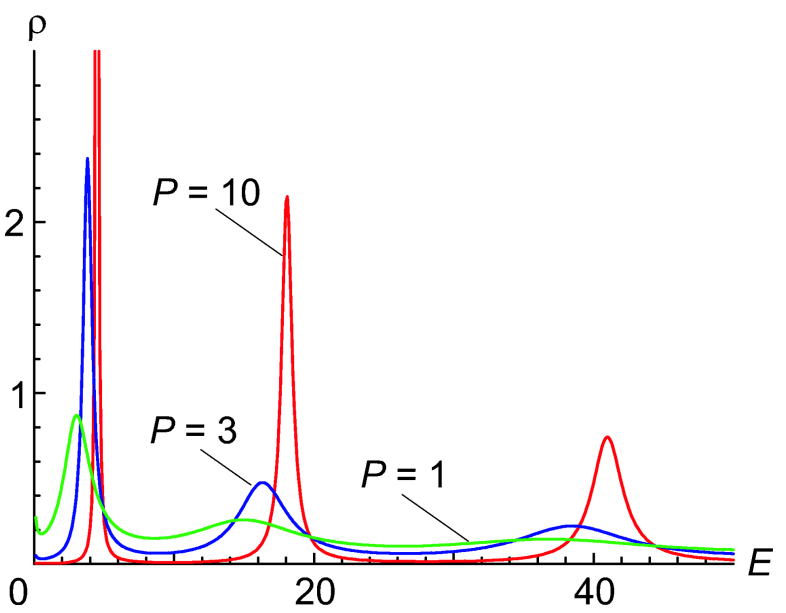

Fig. 11. Local density of states in quantum dot.

When the electron spatial distribution is of interest, the local density of states

$$
\rho(\varepsilon, x)=\sum_{n} \delta\left(\varepsilon-E_{n}\right)\left|\Psi_{n}(x)\right|^{2}
$$

is used instead. Integral version of this definition reads:

$$
\begin{aligned}
\rho(\varepsilon, x) & =\frac{L}{\pi} \int \mathrm{d} k \delta\{\varepsilon-E(k)\}|\Psi(x)|^{2} \\
& =\frac{L}{\pi}\left(\frac{\mathrm{d} \varepsilon}{\mathrm{d} k}\right)^{-1}|\Psi(x \mid \varepsilon)|^{2} .
\end{aligned}
$$

Integrating it over the region $I$ we obtain the density of states in the quantum dot:

$$
\rho_{\mathrm{dot}}(\varepsilon)=\frac{L}{\pi}\left(\frac{\mathrm{d} \varepsilon}{\mathrm{d} k}\right)^{-1} \int_{0}^{1}|\Psi(x \mid \varepsilon)|^{2} \mathrm{~d} x .
$$

This quantity is useful for description of quantum dot properties, such as the tunnelling current directed perpendicular to the dot, measured using scanning tunnelling microscopy (STM) or power absorption in nearfield spectroscopy $[7,8]$.

Replacing the wave function by the eigenfunction used in the derivation of exact solution (A5) with normalization coefficient (A11) we obtain the following expression of local density of states in the decaying quantum dot:

$$
\rho_{\mathrm{dot}}(\varepsilon)=\frac{1}{\pi k Q(k)}\left\{1-\frac{\sin (2 k)}{2 k}\right\},
$$

which is shown in Fig. 11. It is evident that if the zeroes of function $Q(k)$ are close to the real axis (as they are in the case of large $P$ values), they determine the local density of states in the vicinity of the quasibound state leading to the standard Lorentzians. Consequently, the position and the width of the resonant state are described by the same function $Q(k)$ which appears either in exact solution or in the complex energy method.

\section{Quasi-classical approach}

Finally, some words about the simplest quasi-classical method, which was used by Gamow in his theory of radioactive decay. In this case we have to calculate the probability of electron to tunnel through the $\delta$-barrier. It reads:

$$
W=\frac{k^{2}}{k^{2}+P^{2}} \approx \frac{k^{2}}{P^{2}} .
$$

Multiplying this quantity by the frequency of the classical electron reaching the quantum dot edge we obtain the approximate probability for electron to escape from the dot:

$$
\frac{v}{S} W=\frac{k \cdot k^{2}}{2 \cdot P^{2}}=\frac{k^{3}}{2 P^{2}} .
$$

It coincides with the width of the quasi-bound state defined from the approximate Eq. (16).

\section{Conclusions}

The comparison of the exact solution with those obtained by approximate methods enables us to make the following conclusions.

The first pole approximation is quite sufficient for the calculation of the average properties of the quasibound state in the quantum dot. That is why various approximate methods can be used, and they lead to the equivalent results, because most of them are based on the calculation of that pole.

The wave function of emitted electron from the dot is a much more delicate quantity. It is rather sensitive to the boundary conditions at the dot edge, and to the initial condition, due to which the other quantum dot states might be excited. That is why the above wave function of emitted electron demonstrates the emission pulses from those excited states moving with different velocities, and the interference of excited state wave functions in the dot as well. In order to calculate these peculiar wave function features the poles and saddle point contribution have to be properly taken into account.

\section{Acknowledgement}

This work was partly supported by the Lithuanian Science Council Student Research Fellowship Award (G. K.). 


\section{Appendix}

\section{Solution of non-stationary Schrödinger equation}

In this appendix, we present the simple derivation of Eq. (2) solution based on its expansion into the series of some stationary problem eigenfunctions. It can be done by means of the following artificial procedure. We add one more hard wall to the potential at the point $x=$ $L+1$, shown in Fig. 1 by the dotted light (blue on-line) curve, and the corresponding boundary condition

$$
\Psi(L+1, t)=0 .
$$

Now we have the finite system, and its wave function can be obtained applying the standard expansion into the series of eigenfunctions

$$
\Psi(x, t)=\sum_{n} c_{n} \mathrm{e}^{-\mathrm{i} \varepsilon_{n} t} \psi_{n}(x)
$$

with the coefficients calculated as overlap integrals of eigenfunctions and initial function (5),

$$
c_{n}=\int_{0}^{L+1} \mathrm{~d} x \Phi(x) \psi_{n}(x) \equiv \int_{0}^{1} \mathrm{~d} x \Phi(x) \psi_{n}(x) .
$$

The eigenfunctions follow from stationary Schrödinger equation

$$
\left\{H-\varepsilon_{n}\right\} \psi_{n}(x)=0
$$

with hard wall boundary conditions (4) and (A1). Solving the above equation in $I$ and $I I$ regions we obtain the following function:

$$
\psi_{n}(x)=\left\{\begin{array}{l}
A \sin \left(k_{n} x\right), 0<x<1, \\
B \sin \left[k_{n}(x-L-1)\right], \quad 1<x<L+1,
\end{array}\right.
$$

satisfying automatically the above boundary conditions. Here the electron momentum is defined as $k_{n}=$ $\sqrt{2 \varepsilon_{n}}$. Now replacing the Dirac function in Hamiltonian (3) by the corresponding boundary conditions

$$
\begin{aligned}
\psi_{n}^{(I)}(1) & =\psi_{n}^{(I I)}(1), \\
\frac{\mathrm{d}}{\mathrm{d} x} \psi_{n}^{(I I)}(1)-\frac{\mathrm{d}}{\mathrm{d} x} \psi_{n}^{(I)}(1) & =2 P \psi_{n}^{(I)}(1),
\end{aligned}
$$

we arrive at the following set of equations for the coefficients:

$$
\begin{gathered}
A \sin k_{n}=-B \sin \left(k_{n} L\right), \\
A D_{n}=B \cos \left(k_{n} L\right),
\end{gathered}
$$

where

$$
D_{n} \equiv D\left(k_{n}\right), \quad D(k)=\cos k+\frac{2 P}{k} \sin k .
$$

Equating the determinant to zero we obtain the dispersion equation

$$
\tan (k L)=-\frac{1}{D(k)} \sin k,
$$

the solution of which gives us the electron momentum $k_{n}$ and finally the eigenvalue $\varepsilon_{n}$.

Having in mind the wave function normalization and the limit $L \rightarrow \infty$ we obtain the coefficient

$$
B=\sqrt{\frac{2}{L}} .
$$

Then the coefficient $A$ follows from Eqs. (A7a) and (A9):

$$
\begin{aligned}
A & =-B \frac{\sin \left(k_{n} L\right)}{\sin k_{n}}=\frac{\sqrt{2 / L} \tan \left(k_{n} L\right)}{\sin k_{n} \sqrt{1+\tan ^{2}\left(k_{n} L\right)}} \\
& =\sqrt{\frac{2}{L Q\left(k_{n}\right)}} .
\end{aligned}
$$

Now we calculate integral (A3)

$$
\begin{aligned}
\frac{c_{n}}{A} & =\sqrt{2} \int_{0}^{1} \mathrm{~d} x \sin \left(k_{n} x\right) \sin (\pi x) \\
& =\frac{\sqrt{2} \pi \sin k}{\left(\pi^{2}-k^{2}\right)} \equiv F(k)
\end{aligned}
$$

and present the solution of our non-stationary problem (2) as

$$
\begin{aligned}
& \Psi(x, t)=\frac{2}{L} \sum_{n} \frac{F\left(k_{n}\right)}{Q\left(k_{n}\right)} \mathrm{e}^{-\mathrm{i} \varepsilon_{n} t} \\
& \times\left\{\begin{array}{l}
\sin \left(k_{n} x\right), \quad x<1, \\
\sqrt{Q\left(k_{n}\right)} \sin \left[k_{n}(x-1-L)\right], \quad x>1 .
\end{array}\right.
\end{aligned}
$$

We have to perform one more step, namely, to calculate the limit $L \rightarrow \infty$. We see that the dispersion Eq. (A9) is composed of the rapidly varying function $\tan (k L)$ equated to the slowly varying one. Consequently, in the case of large $L$ the distance between eigenvalues is $\Delta k=\pi / L$ and this enables us to calculate the above limit using just the formal replacement

$$
k_{n} \rightarrow k \text { and } \quad \sum_{n} \rightarrow \frac{L}{\pi} \int_{0}^{\infty} \mathrm{d} k
$$


The only thing that should be taken into account is the wave function (A13) dependence on large value $L$. It can be eliminated using the trigonometric expression

$$
\begin{aligned}
\sin [k(x-1-L)]= & \sin [k(x-1)] \cos (k L) \\
& -\sin (k L) \cos [k(x-1)]
\end{aligned}
$$

and having in mind that

$$
\sin (k L)=-\frac{\sin k}{\sqrt{Q(k)}}, \quad \cos (k L)=\frac{D(k)}{\sqrt{Q(k)}},
$$

what follows from Eq. (A9).

Thus, performing the limit $L \rightarrow \infty$ and using above equations we immediately arrive at the final wave function Eq. (6).

\section{References}

[1] T. Chakraborty, Quantum Dots (Elsevier, Amsterdam, 1999).

[2] K.S. Novoselov, A.K. Geim, S.V. Morozov, D. Jiang, M.I. Katsnelson, I.V. Grigorieva, S.V. Dubonos, and A.A. Firsov, Nature (London) 438, 197 (2005).

[3] Y. Zang, Y.W. Tan, H.L. Stormer, and P. Kim, Nature (London) 438, 201 (2005).
[4] M.I. Katsnelson, K.S. Novoselov, and A.K. Geim, Nature Phys. 2, 620 (2006).

[5] P. Hawageegana and V. Apalkov, Phys. Rev. B 77, 245426 (2008).

[6] M.L. Goldberger and K.M. Watson, Collision Theory (Wiley, New York, 1964).

[7] A. Matulis and F.M. Peeters, Phys. Rev. 77, 115423 (2008).

[8] M.R. Masir, A. Matulis, and F.M. Peeters, Phys. Rev. 79, 155451 (2009).

[9] Hong-Yi Chen, V. Apalkov, and T. Chakraborty, Phys. Rev. Lett. 98, 186803 (2007).

[10] J. Petzold, Z. Phys. 155, 422 (1959); R.G. Winter, Phys. Rev. 123, 1503 (1961).

[11] W. van Dijk, F. Kataoka, and Y. Nogami, J. Phys. A 32, 6347 (1999).

[12] W. van Dijk and Y. Nogami, Phys. Rev. Lett. 83, 2867 (1999).

[13] W. van Dijk and Y. Nogami, Phys. Rev. C 65, 024608 (2002).

[14] G. García-Calderón, J.L. Mateos, and M. Moshinsky, Phys. Rev. Lett. 74, 337 (1995).

[15] A. Sommerfeld, Partial Differential Equations in Physics: Lectures on Theoretical Physics, vol. 6 (Academic Press, 1964).

\title{
ARTUTINIS SKYLANČIOS KVAZISTACIONARIOSIOS BŪSENOS APRAŠYMAS
}

\author{
A. Matulis ${ }^{a}$, G. Kiršanskas ${ }^{b}$ \\ a Puslaidininkiu fizikos institutas, Vilnius, Lietuva \\ ${ }^{\mathrm{b}}$ Vilniaus universitetas, Vilnius, Lietuva
}

\section{Santrauka}

Nagrinejjamas paprastas skylančios kvantinès būsenos modelis, kuriame vienmatis elektronas juda potenciale, susidedančiame iš Dirako smaile atskirto kvantinio taško nuo begalinès teigiamos $x$ pusašès. Kvantinio taško charakteristikos (kvazistacionaraus lygmens energija bei gyvavimo trukmè) ir išspinduliuoto elektrono banginė funkcija, apskaičiuoti artutiniais kompleksinès energijos bei lokalaus lygmenų tankio metodais, palyginami su tiksliu analiziniu sprendiniu, išreikštu kontūriniu integralu kompleksinejje elektrono impulso plokštumoje. Aptariamos fizikinès išspinduliuoto elektrono banginès funkcijos ypatumų priežastys. 